(35) J. Evans, $A p . J$. 120, 94, 1954.

(36) J. Ramberg, Stockh. Obs. Ann. 17, No. 2, 195I; H. Kristenson, ibid. 18, No. 5, I955.

(37) A. Colacevich, Atti del Convegno Volta, r953.

(38) M. N. Gnevyshev, C.R. Acad. Sci. U.R.S.S. 94, 8 I I, I954.

(39) W. O. Roberts et al., Astr. J. 58, 225, 1953 (and in the Press).

(40) A. Bruzek, $Z$. Naturf. 7 a, 708, 1952.

(4I) A. Bruzek, $Z$. Ap. (in the Press).

(42) M. Notuki and I. Shimizu, Tokyo Observatory Report, 10, 258, 1953.

(43) C. W. Allen, M.N. 106, I36, 1946.

\title{
APPENDIX
}

\section{REPORT OF THE WORKING GROUP ON FLARE CLASSIFICATION}

During the Eighth General Assembly of the International Astronomical Union held at Rome in September I952, it was resolved to appoint a Working Group of Commission II to report on improved methods of flare classification. The members of this committee were: L. d'Azambuja, Marguerite d'Azambuja, Helen Dodson, M. A. Ellison (chairman), H. W. Newton and A. Severny. A great volume of observational material has had to be analysed in the preparation of this report, and I acknowledge with gratitude the generous and indispensable co-operation of these individuals, as well as that of Dr P. A. Wayman of Herstmonceux.

Twenty years have elapsed since it was planned to organize the observation of solar flares on an international basis, under the auspices of the I.A.U.(r). During this period flares have been classified on a scale of importance-I (smallest), 2 (intermediate), 3 (largest); the further category $3+$ (introduced by Newton) is also in general use to denote flares of outstanding importance. In addition, many observers use intermediate steps, such as I + or $2-$ to indicate their estimate that the flare exceeds importance I but is less than 2. Although it was recommended in I935 that estimates should be based upon area alone, the scale of importance has remained very largely a subjective scale: it has always been left to the discretion of individual observers to decide to what extent their estimates of importance should be based upon quantitative measures.

Consequently, the essential problem before the committee has been to review the existing situation and to decide:(a) how many steps in the importance scale are desirable, (b) to what extent, and in what way, the estimates should be based upon quantitative measures, such as area, line-width or central intensity of the $\mathrm{H} x$ emission, and $(c)$ to provide simple guidance in these matters for observers who use different instruments and different methods of observation.

In the early days, flare patrols were carried out visually by means of spectrohelioscopes, usually of the Hale pattern, and this method is still widely employed. More recently, automatic spectroheliographs and Lyot filters employing cinematography have been introduced. The present multiplicity of instruments increases the difficulties of our problem, since, for example, the measured central intensities of $\mathrm{H} \alpha$ in a flare stand in need of considerable correction, and these corrections can only be applied if the constants of each instrument have been determined. The corrections are a function both of the instrument and of the brightness of the flare.

The quantities amenable to measurement are as follows:

(a) duration of the flare from start to finish;

(b) area of the region, or regions, of enhanced emission in $\mathrm{H} \alpha$;

(c) effective line-width of $\mathrm{H} \alpha$ in angstroms at the brightest point;

(d) the central intensity of $\mathrm{H} \alpha$, expressed as a fraction of the level of the continuous spectrum outside the line, or in terms of the background intensity of the Fraunhofer $\mathrm{H} \alpha$.

Of these, $(d)$ is perhaps the most difficult to determine because of the short duration of maximum intensity and of the uncertainty of the instrumental corrections involved.

$$
146
$$




\section{Duration}

We consider that suddenness of commencement is one of the main criteria for distinguishing between flares and those slower variations in brightness of the plage areas where flares are located. Flare development curves (line-widths or central intensities plotted versus time) show many different rates of rise and decline, but in any individual case the rate of rise always exceeds the rate of decay. For these reasons the time of commencement of a flare may usually be fixed within I-2 min., whereas the time of ending, owing to the slower rate of decay and the uncertainty of what was the preflare brightness of the region, cannot be given with equal precision.

Nevertheless, there is general agreement among observers as to the duration of flares which have been fully observed. The mean durations shown in Table I may, therefore, be adopted as a guide. We can see, from the figures of the last column, that a wide range of durations is found in any one importance class.

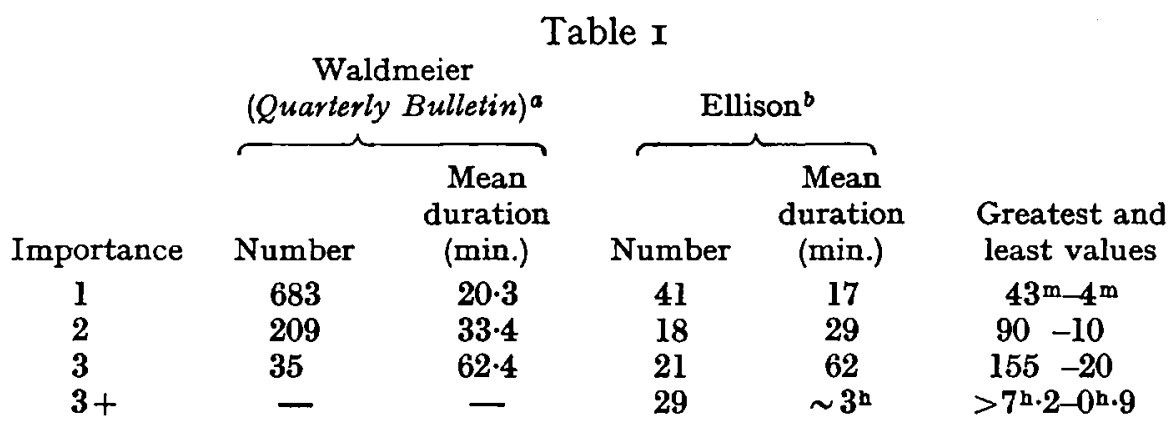

a M. Waldmeier. Statistische Untersuchungen an chromosphärischen Eruptionen. Astronomische Mitteilungen der eidgenössischen Sternwarte Zürich, Nr. I53, 1948.

b M. A. Ellison. Characteristic Properties of Chromospheric Flares. M.N.R.A.S. 109, 3, r949.

\section{Area measurements}

The available measures of area are given in Table 2 ; they relate to the times of maximum brightness of the flares and are expressed in the unit $10^{-6}$ of the visible hemisphere. The figures show marked differences between different observers, as is only to be expected in the absence of any generally recognized relationship between importance and area.

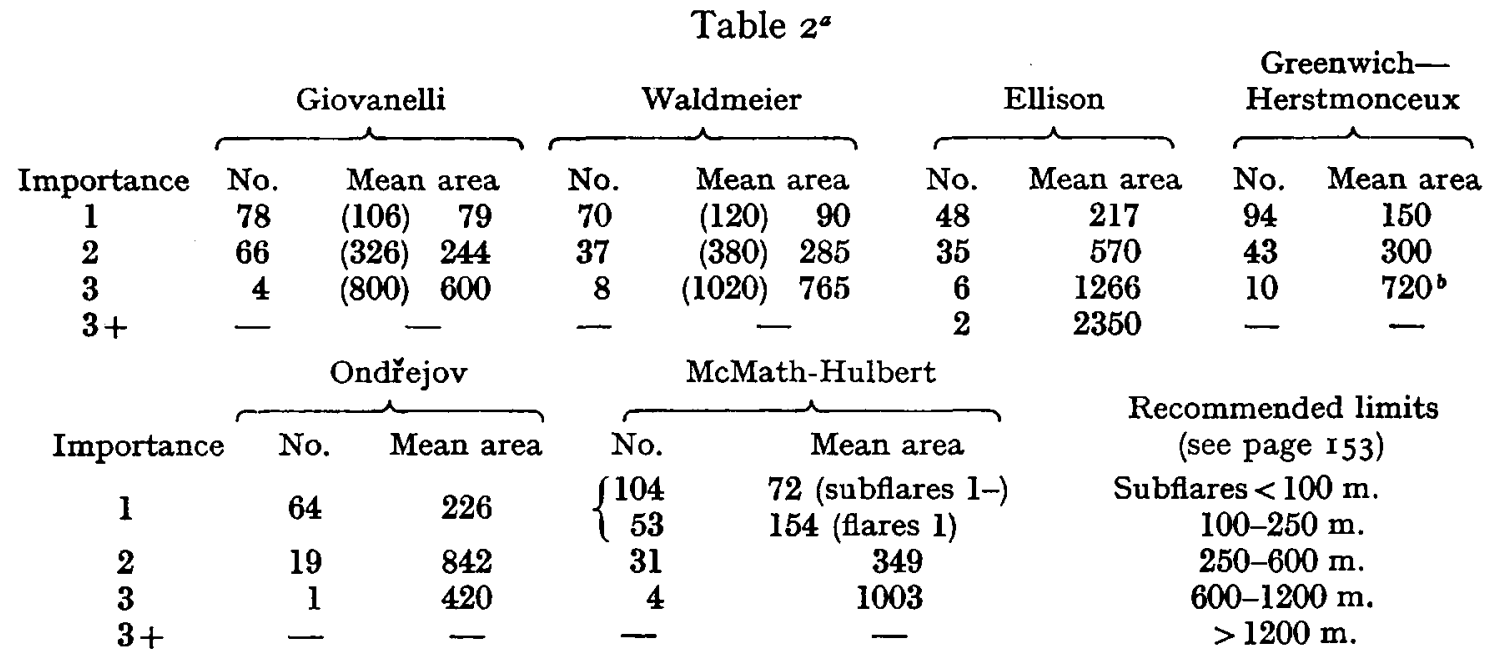

a The mean areas in this table are all expressed in terms of the unit $10^{-6}$ of the visible hemisphere. The figures within brackets are the published values expressed in the unit $10^{-6}$ of solar disk (correction factor taken as 0.75).

b Includes two flares $3+$ with areas 950 and 1200 millionths. 
We find that it is the current practice of many observers to base their estimates of importance primarily upon the corrected area.* Thus, for example, at Meudon and Edinburgh the limits of area (corrected) given in Table 3 are employed for classification purposes:

\begin{tabular}{ccccc} 
& \multicolumn{2}{c}{ Meudon } & Table 3 & \multicolumn{2}{c}{ Edinburgh } \\
Importance & Square degrees & Millionths & $\overbrace{\text { Square degrees }}^{\text {Millionths }}$ \\
1 & $<5-6$ & $<242-291$ & $2 \cdot 06-6 \cdot 18$ & $100-300$ \\
2 & $>5<15$ & $>242<727$ & $6 \cdot 18-15 \cdot 5$ & $300-750$ \\
3 & $>15$ & $>727$ & $15 \cdot 5-24 \cdot 7$ & $750-1200$ \\
$3+$ & $>15$ & $>727$ & $>24 \cdot 7$ & $>1200$
\end{tabular}

In the Edinburgh scheme of classification there is the further provision that a flare must be rated above, or below, the importance corresponding to its corrected area, if it exhibits a maximum intensity, or an effective line-width, considerably greater, or less, than that which experience shows is normal for the class. We recommend that this method of procedure should be generally adopted in accordance with the guiding principles set out on page $\mathrm{I}_{53}$.

There remains the question whether to adopt for classification purposes the areas as measured (fractions of the disk), or the areas as corrected for foreshortening (fractions of the hemisphere). The latter are obtained from the former by use of the factor sec $h$, where $h$ is the heliocentric angle. The correction is valid only if the emission region of a flare lies in a plane parallel to the solar surface and has a vertical thickness small in comparison to its horizontal extension. Information is inadequate; for the same flare cannot be observed both in plan and elevation. The greatest $3+$ flares undoubtedly have a larger projected area when seen near the centre of the disk than near the limb. There is also the evidence derived from Miss Dodson's analysis of the variations in average line-width between centre and limb (Table 6), which points to flares having a greater optical depth the further they are from the central meridian. On balance, therefore, we are of the opinion that corrected areas should be generally adopted, and we feel that the errors introduced by failing to use the sec $h$ correction would be greater than if we were to use measured areas alone. Correction may tend to overrate flares near the limb, but in any event classification of limb flares is bound to remain uncertain in the present state of our knowledge.

The measured areas should refer to the time of maximum brightness (or line-width) of the main emission region. The area usually increases after maximum brightness, as the emission slowly spreads while its intensity diminishes. With a spectrohelioscope, when a flare is observed from the start, an experienced observer can distinguish without difficulty between areas of enhanced emission and neighbouring parts of the plage which have not increased in brightness. Experiments made at Edinburgh show that different observers, measuring the same flare, will usually agree to within $20 \%$. However, Dr Wayman's analysis shows that when the same flare has been observed at two or more different observatories, discrepancies much greater than this are common. Here is a strong argument for emphasizing the importance of the area measurements, for improving their precision and for achieving uniformity in the matter of correction along the

* For measurements of area with the spectrohelioscope, a suitable graticule may be photographed on glass, having squares of side $0 \cdot 0{ }_{75} R$ (i.e. $\mathrm{I}^{\circ}$ ), where $R$ is the mean radius of the solar image. The graticule is placed in close proximity to the first or second slit. Then we have

$$
A=A_{m} \cdot \sec h \cdot 4^{8 \cdot 5} \text {, }
$$

where $A$ is the corrected area in millionths of the hemisphere, $A_{m}$ is the measured (i.e. projected) area in square degrees, $h$ is the heliocentric angle of the flare, and the factor $4^{8.5}$ is the number of millionths of the hemisphere contained in I square degree at the disk centre. 
lines mentioned above. It may prove that small-scale photographic images lead to over-estimates of area due to halation and spreading in the emulsion; this is a difficult photometric problem and should be investigated for each instrument.

\section{Effective line-widths}

Such measurements have been made by individual workers with spectrohelioscopes over the past I5 years (2), but the results have been included in the Quarterly Bulletin only since I January I949. Miss Dodson, who has made a statistical analysis of the linewidth measures appearing in the Quarterly Bulletin (I January I949-30 June I952), gives the averages shown in Table 4:

Table 4

Importance
$\mathbf{1}$
$\mathbf{2}$
$\mathbf{3}$

$\begin{array}{cc}\text { Table } 4 & \begin{array}{c}\text { Average width of } \mathrm{H} \alpha \text { in } \AA \\ \text { at flare maximum }\end{array} \\ \text { No. of cases } & 2 \cdot 71 \\ 457^{a} & 3.91 \\ 139 & 6 \cdot 98 \\ 14 & \\ \text { Total } 610^{a} & \end{array}$

a Includes two cases of uncertain importance.

This table clearly brings out the well-established dependence of maximum line-width upon importance. However, the maximum line-width is of extremely short durationoften only a matter of seconds. Experience shows that unless an observer makes a continuous plot of line-width versus time, taking readings at least as frequently as one every half-minute, he will almost certainly miss the peak value. We have, therefore, examined the maximum values derived from the line-width development curves obtained at Ondłejov by Link and Mášková (3), and at Edinburgh, in which there can be no doubt that the actual maxima were recorded. These results, shown in Table 5, give somewhat higher values for the average line-width at maximum, and the agreement between the two observatories is seen to be good for flares of importance $I$ and 2 for which the data are sufficiently numerous.

\begin{tabular}{|c|c|c|c|c|c|c|}
\hline & & OndY̌ejov & & & Edinburgh & \\
\hline Importance & No. & $\begin{array}{l}\text { Av. line-width } \\
\text { at max. from } \\
\text { development } \\
\text { curves } \\
(\AA)\end{array}$ & $\begin{array}{c}\text { Extremes } \\
(\AA)\end{array}$ & No. & $\begin{array}{l}\text { Av. line-width } \\
\text { at max. from } \\
\text { development } \\
\text { curves } \\
(\AA)\end{array}$ & $\begin{array}{c}\text { Extremes } \\
(\AA)\end{array}$ \\
\hline 1 & 132 & $\mathbf{3} \cdot 0$ & $1 \cdot 8-7 \cdot 1$ & 48 & $3 \cdot 2$ & $1.9-8.8$ \\
\hline 2 & 31 & $5 \cdot 1$ & $2 \cdot 5-11 \cdot 2$ & 21 & $4 \cdot 9$ & $2 \cdot 5-9 \cdot 7$ \\
\hline 3 & 2 & $7 \cdot 0$ & $6 \cdot 9-7 \cdot 1$ & 7 & $11 \cdot 0$ & $2 \cdot 6-22 \cdot 9$ \\
\hline
\end{tabular}

While there is no doubt that the average line-width at maximum increases with flare importance, the use of these measures for classification purposes is somewhat complicated by the fact that the line-width is, likewise, a function of the heliocentric angle. This is clearly brought out by Miss Dodson's analysis of the Quarterly Bulletin measures (Table 6) which she has separated out according to central meridian distance.

The interpretation of these results is by no means clear at present. They may well indicate a change in optical depth of the flare emission regions which is dependent upon the angle of vision (4), but it is also probable that variations in the profile of the Fraunhofer $\mathrm{H} \alpha$ line between centre of disk and limb (in relation to which the widths of the emission lines are measured) are also involved. 
Miss Dodson has further demonstrated that the total number of flares observed decreases as we move from the central meridian towards the limb, the fall in numbers being particularly marked as we pass beyond $60^{\circ}$.

In view of these facts, it must be emphasized that, while line-widths constitute a valuable guide for classification purposes, they should be regarded as of secondary importance to areas; they should be used with due caution and on the basis of the data given in Tables $4-6$.

Table 6

\begin{tabular}{|c|c|c|c|c|c|}
\hline \multirow{3}{*}{$\begin{array}{c}\text { Central } \\
\text { meridian } \\
\text { distance } \\
0^{\circ}\end{array}$} & \multicolumn{5}{|c|}{ Average width of $H \propto$ at maximum } \\
\hline & Flares, & imp. 1 & Imps. 2 and 3 & To & \\
\hline & $2 \cdot 48$ & (5) & $-\quad(0)$ & $2 \cdot 48$ & (5) \\
\hline $1^{\circ}-10^{\circ}$ & $2 \cdot 45$ & (68) & $3 \cdot 66$ & $2 \cdot 71$ & (86) \\
\hline $11^{\circ}-20^{\circ}$ & $2 \cdot 53$ & (54) & $3 \cdot 29$ & $2 \cdot 69$ & (69) \\
\hline $21^{\circ}-30^{\circ}$ & $2 \cdot 56$ & (46) & $3 \cdot 49$ & $2 \cdot 87$ & (69) \\
\hline $31^{\circ}-40^{\circ}$ & $2 \cdot 60$ & (65) & $4 \cdot 12$ & $2 \cdot 91$ & (82) \\
\hline $41^{\circ}-50^{\circ}$ & $2 \cdot 73$ & (53) & $4 \cdot 03$ & $3 \cdot 17$ & (80) \\
\hline $51^{\circ}-60^{\circ}$ & $2 \cdot 80$ & $(67)^{a}$ & $4 \cdot 69$ & $\mathbf{3} \cdot 30$ & $(91)^{a}$ \\
\hline $61^{\circ}-70^{\circ}$ & 2.99 & $(56)^{a}$ & $5 \cdot 24$ & $\mathbf{3 \cdot 4 7}$ & $(71)^{a}$ \\
\hline $71^{\circ}-80^{\circ}$ & $2 \cdot 89$ & (31) & $6 \cdot 00$ & $3 \cdot 59$ & (40) \\
\hline $81^{\circ}-90^{\circ}$ & $3 \cdot 80$ & (12) & $4 \cdot 24$ & 3.93 & (17) \\
\hline Total & $2 \cdot 71$ & $(457)^{b}$ & $4 \cdot 19(153)$ & 3.08 & 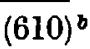 \\
\hline
\end{tabular}

a Includes one case of uncertain importance. $\quad b$ Includes two cases of uncertain importance.

A line-width at maximum of $2 \cdot 0 \AA$ may be taken as about the lowest value met with in Class I flares. Conversely, the greatest $3+$ flares have exceptionally wide emission lines, wherever they are located on the disk. We give four examples:

\begin{tabular}{|c|c|c|c|c|}
\hline \multicolumn{2}{|c|}{ Date } & Importance & $\begin{array}{l}\text { Line-width at } \\
\text { maximum }(\AA)\end{array}$ & C.M. distance $\left({ }^{\circ}\right)$ \\
\hline 1946 & July 25 & $3+$ & 16 & 15 \\
\hline 1949 & Feb. 11 & $3+$ & 16 & 77 \\
\hline 1949 & Sept. 18 & $3+$ & 12 & 69 \\
\hline 1949 & Nov. 19 & $3+$ & 23 & 70 \\
\hline
\end{tabular}

\section{Photometry of central intensities}

The emission $\mathrm{H} \alpha$ line of a flare is normally observed against the background of the Fraunhofer $\mathrm{H} \alpha$ line (full lines of Fig. I).

The contrast (visual or photographic) between the emission and its background, therefore, alters with the setting in wave-length of the selecting, or viewing slit, being normally at a maximum in position $\mathrm{I}$ and zero in positions 2 and $3 . X Y$ is the visibility range, or effective line-width expressed in arigstroms: $A B / B I$ is the central intensity expressed as a fraction of the continuum: $A B \mid B C$ is the central intensity in terms of the chromospheric background.

The observed profiles (full lines) stand in need of correction to give true profiles (dashed lines). The chief corrections to be applied (s) are for:

(a) scattered heterochromatic light;

(b) grating ghosts;

(c) finite widths of slits.

Of these $(a)$ is by far the most serious. In a good spectrograph the contributions of $(a),(b)$ and $(c)$ will not exceed $20 \%\left(C C^{\prime}\right)$ of the continuum. Thus, for example, with the Edinburgh spectrograph, the observed central intensity of Fraunhofer $\mathrm{H} \alpha$ is $24 \%$, which reduces after correction to $16 \%$. 
So far as the corrections to the central intensity of the flare emission lines are concerned, these are, broadly speaking, such as to increase the observed c.r.'s when the intensity exceeds the continuum, and to reduce them when the intensity is inferior to the continuum. In the neighbourhood of the continuum level the corrections are small, or vanish. Without correction, we should, therefore, expect the photometric measures of the less intense (Class I) flares to be the most reliable. The method of correction has not yet been investigated in detail for spectroheliographs or for Lyot filters. It may be noted that effective line-width $(X Y)$ is virtually unaffected by these corrections to the profiles.

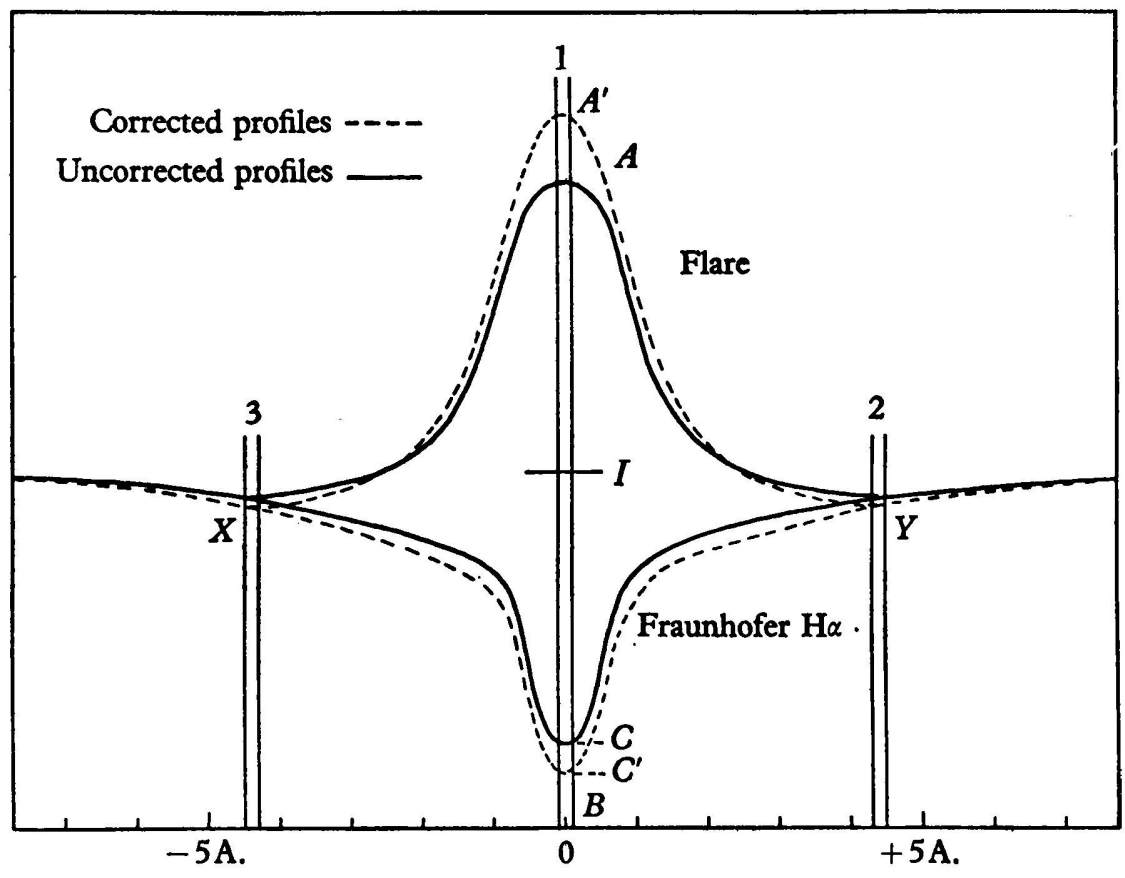

Fig. I

For these reasons we have at present a number of 'local' scales of central intensity versus importance which cannot be reconciled until the magnitudes of the corrections have been more fully investigated in each case. The number of measures available is also inadequate, though it would appear that these will soon become more numerous with the increasing use of automatic spectroheliographs and Lyot type $\mathrm{H} \alpha$ heliographs.

In Table 7 are summarized the results of $(a)$ a long series of measurements made (I936-46) with the Greenwich spectrohelioscope (measurements corrected for scattered light and referring to time of maximum), (b) Miss Dodson's results obtained with the McMath-Hulbert automatic spectroheliograph (referring to time of maximum and corrected for heterochromatic scattered light), (c) Arcetri measurements from calibrated spectroheliograms (I949-52), and (d) a small number of measurements from Mitaka.

Table 7

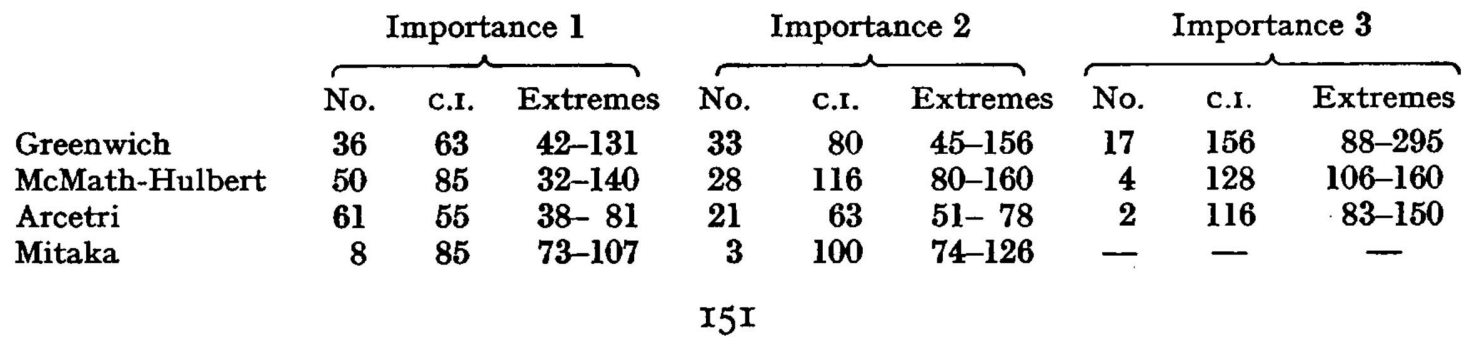


The Edinburgh measurements made with spectrograph and spectrohelioscope suggest considerably higher values for Class 2 and Class 3 flares at the time of maximum (5).

Edinburgh, McMath-Hulbert and Meudon, however, are in agreement that a sudden brightening should reach a C.I. of at least $80 \%$ of the continuum to be rated as a Class I flare. Miss Dodson's plot of C.I. versus flare area (Fig. 2) shows that flares of this intensity at maximum have an average area of about roo $\mathrm{m}$.

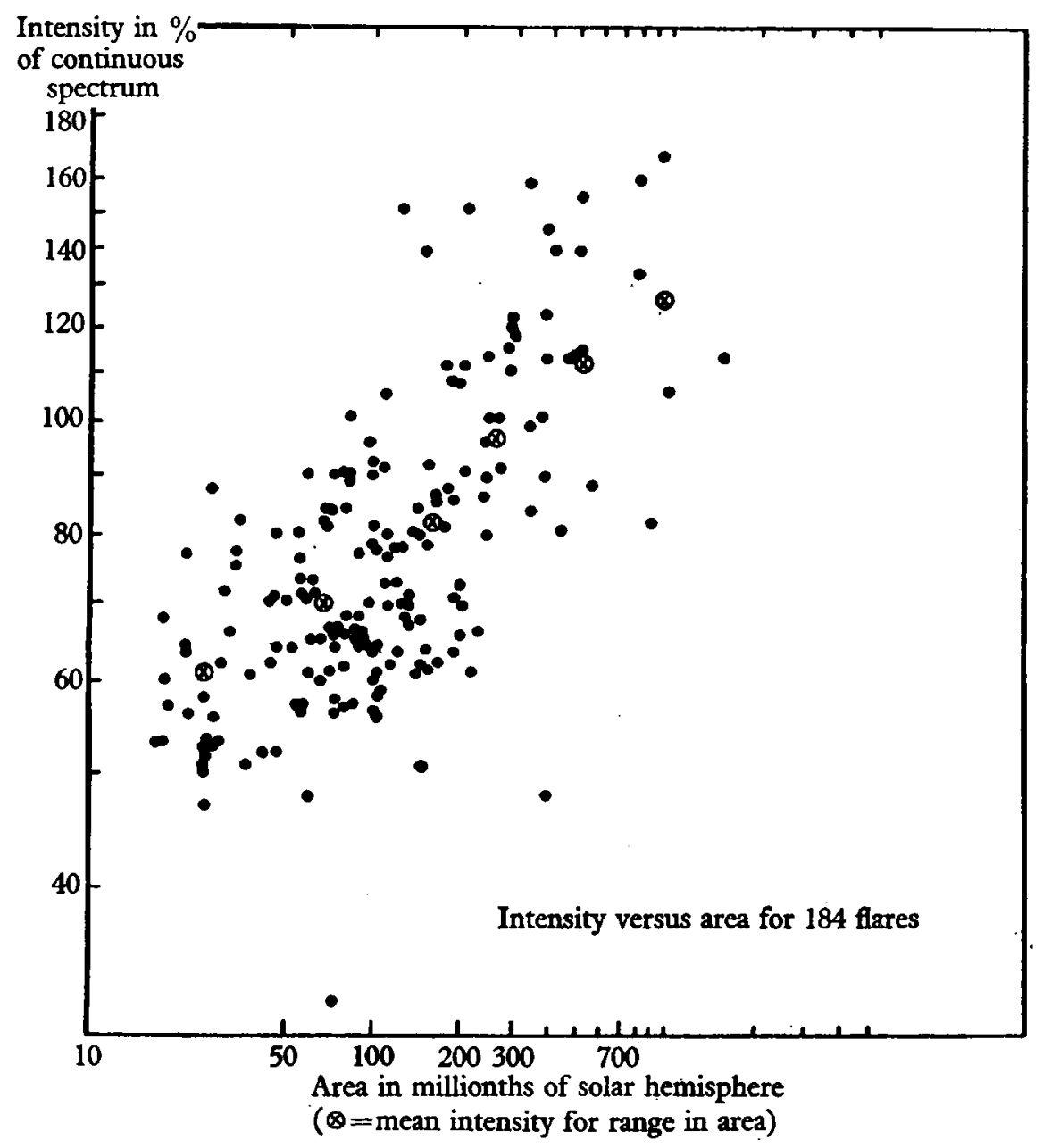

Fig. 2

\section{Recommendations}

r. We would first draw attention to the immense complexity of the flare phenomenon. This is evident when we consider flares as the source points of ultra-violet light, surge prominences, auroral particles, cosmic rays and radio noise. It is unlikely that any single code number, based solely upon observations in one wave-length $(\mathrm{H} \alpha)$ will adequately represent a phenomenon whose physical properties are so incompletely understood. In these circumstances we wish to emphasize the remarkable accord (rather than the discrepancies) and the high value of the flare classifications and measurements which have been made during the past 20 years.

2. We wish to stress the need for more and better measurements of area, line-width and central intensity. 
3. We recommend that area should be used as the primary basis for classification by importance. The corrected area, measured at the time of maximum brightness, should be used and it should be expressed in millionths of the visible hemisphere or in square degrees (I sq. degree at the centre of the disk $=48.5 \mathrm{~m}$.). We recommend that the following limits of area should be adopted:

\begin{tabular}{lccc} 
& Importance class & \multicolumn{2}{c}{ Corrected area } \\
Subflares & $1-$ & $<100 \mathrm{~m}$. & $<2 \cdot 06$ sq. degrees \\
Flares & 1 & $100-250$ & $2 \cdot 06-5 \cdot 15$ \\
Flares & $\mathbf{2}$ & $250-600$ & $\mathbf{5} \cdot \mathbf{1 5}-\mathbf{1 2} \cdot 4$ \\
Flares & $\mathbf{3}$ & $600-1200$ & $\mathbf{1 2 \cdot 4}-\mathbf{2 4} \cdot \mathbf{7}$ \\
Flares & $\mathbf{3}+$ & $>1200$ & $>\mathbf{2 4} \cdot \mathbf{7}$
\end{tabular}

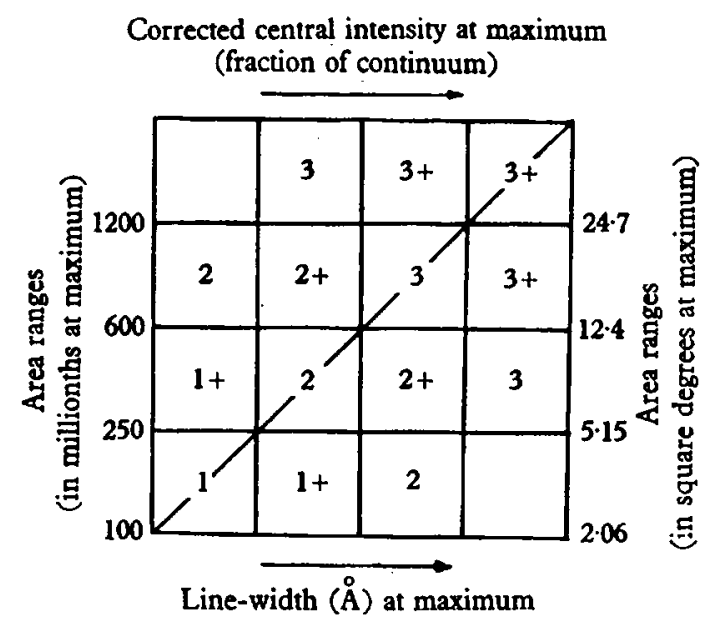

Fig. 3

We introduce the new class subflare $\left(\mathrm{I}^{-}\right)$to denote sudden brightenings in plage regions whose areas are inferior to roo $\mathrm{m}$. These are perhaps the most numerous of all flares, but their geophysical effects are probably negligible. We are of the opinion that subflares of area $<50 \mathrm{~m}$. need not be recorded or reported to the Quarterly Bulletin. Their visibility is determined to a large extent by instrumental and seeing conditions.

4. We also recognize, from our analysis of data, that in any one importance class there is a large and real scatter in the values of central intensity and line-width. This has been pointed out by many experienced observers and is clearly seen in Fig. 2.

We therefore recommend that the new scale should have the additional categories I + (i.e. I.5) and $2+$ (i.e. $2 \cdot 5$ ) (see Fig. 3). Thus a flare (class $\mathrm{I}$ ) having an area within the range I0o- $250 \mathrm{~m}$. should be rated $\mathrm{I}+$ if its central intensity of line-width are found to be much greater than the normal values for a class $I$ flare. Similarly, a flare (class 2) having an area within the range $250-600 \mathrm{~m}$. should be rated $\mathrm{I}+$ if its central intensity or line-width is found to be much less, and $2+$ if either of these is much greater than the normal values. Likewise, a subflare, whose area lies within the range 50-roo m., may be elevated to Class I status if it is unusually bright. In judging what are the normal values of central intensity and line-width, observers should continue to rely upon their past experience and consult the data given in the foregoing tables.

We recommend that the ratings such as $2-$ and $3-$, formerly used, should be discontinued.

5. Fig. 3 is presented merely as a guide to observers in deciding the importance of a flare. We have refrained from inserting any precise values of central intensity and linewidth (which increase from left to right along the horizontal axes of the diagram), but we hope that, with the accumulation of information, it may be possible to allot such 
figures by general agreement in the future. Meanwhile, we offer the following statement for the guidance of observers who are in a position to measure central intensities: If an observer knows that the central intensity of a flare at maximum is as much as 0.8 of the continuum, he can feel justified in calling it a flare of importance $I$. If the intensity is greater than the continuous spectrum and the corrected area is of the order of 250 millionths or more, the importance can safely be estimated as at least 2 . No flare should be called importance 3 if its intensity is known to be less than that of the continuous spectrum. Flares for which the intensity is more than twice that of the local continuum are generally of importance 3 or $3+$.

6. It is emphasized that the new scale based on area is closely related to the present practice of most observers, and there will be no appreciable discontinuity between lists of flares drawn up on the new and the old systems. If the new scale is consistently applied, it may lead to considerably better accord than in the past.

7. Geophysical effects of flares (e.g. crochets, fade-outs, s.E.A.'s, etc.) should not be allowed to influence the importance classification. In this way we shall avoid prejudging the question (still to be decided) whether all flares of the same $\mathrm{H} \alpha$ importance have comparable ionospheric effects.

8. Flare surges are an important, and as yet little understood, feature of the flare process. These prominences make their first appearance nearby within a few minutes of the time of peak intensity of the flare emission; when seen against the disk they exhibit dense absorption by contrast with the wings of the $\mathrm{H} \alpha$ line. They are best observed by 'hunting' with the spectrohelioscope line-shifter, since they show a characteristic sequence of sightline velocity-blue shift followed by red shift (6).

In view of their intrinsic interest, and their possible bearing upon the radio noise and cosmic ray phenomena, we recommend that their association with flares should be reported to the Quarterly Bulletin. This can be done by the insertion of the letter $d$ after the figure which denotes the flare importance, and in special cases by an independent note giving the maximum observed sightline velocities.

9. Greater attention should be given to the correct assignment of the co-ordinates of flares. Discrepancies between different observations of the same flare are often considerable. The heliographic latitude and central meridian distance should be stated for the time of flare maximum, and these should refer to the 'centre of gravity' of the emission region, rather than the brightest point.

\section{A. ELLISON \\ Chairman of the Working Group}

\section{REFERENCES}

(I) Bulletin for Character Figures of Solar Phenomena, no. 26 (1934) and no. 3I (1935).

(2) M.N.R.A.S. ro9, 3, 1949 and Publ. R.O. Edinb. 1, 75, 1952 (for definition and method).

(3) F. Link and D. Máśková, B.A.C. 2, I86-r96 and 3, 59-64.

(4) $A p . J$. 120, 83, 1954 .

(5) Publ. Roy. Obs. Edinb. 1, 75, 1952.

(6) M.N.R.A.S. 102, 2, 1942; 102, I1, 1942.

I $a$ a. SUB-COMMISSION ON CINEMATOGRAPHY OF CHROMOSPHERIC PHENOMENA

The principal question facing the sub-commission is that of accomplishing international co-ordination of the cinematography of chromospheric and prominence phenomena. At the r948 meeting of Commission II the goal was set of producing films, by inter-observatory co-operation, presenting in uninterrupted sequence the whole life-evolution of prominence and chromospheric manifestations of active solar regions, not only for the limb but also the disk manifestations of the activity. The increasing number of observatories now engaged in obtaining such films brings the long-established goal within hope of realization. However, serious problems of the magnitude of the task of assembling 